\title{
Nonlinear Predictive Control System for Stiction Compensation in Electropneumatic Control Valves
}

\author{
M. E. U. J. Araújo $\mathbb{D}^{1,2}$ J. R. T. Gadelha ${ }^{1},{ }^{2}$ W. M. Santos $\mathbb{D},^{2}$ \\ A. L. Maitelli, ${ }^{2}$ and F. M. U. Araújo \\ ${ }^{1}$ Department of Informatics, Federal Institute of Paraíba, N-720, 58015-435 João Pessoa, PB, Brazil \\ ${ }^{2}$ Department of Automation and Computing, CT, Federal University of Rio Grande do Norte, 59078-970 Natal, RN, Brazil \\ Correspondence should be addressed to M. E. U. J. Araújo; marcio.ugulino@ifpb.edu.br
}

Received 27 May 2017; Revised 11 January 2018; Accepted 28 January 2018; Published 2 April 2018

Academic Editor: Petko Petkov

Copyright (C) 2018 M. E. U. J. Araújo et al. This is an open access article distributed under the Creative Commons Attribution License, which permits unrestricted use, distribution, and reproduction in any medium, provided the original work is properly cited.

\begin{abstract}
This paper presents the implementation of a system that deals with static friction (stiction) in electropneumatic control valves, one of the most common nonlinearities that causes problems such as limit cycles and consequently wear of the valve and its moving parts, as well as losses in production and maintenance costs. This system is composed of a nonlinear predictive controller with adjustable constraints and an online database for estimation of the stiction parameters. The predictive controller uses constraints on the valve speed during its excursion, as well as constraints on the control signal to bring the valve to the desired position and slip it when necessary. The strategy adopted also showed robustness, being able to cope with changes in the spring and stiction parameters, which caused mismatch between the model and the controller and consequently loss of performance or even instability.
\end{abstract}

\section{Introduction}

Electropneumatic valves are common in the petrochemical area and are essential in many other industrial processes on fluid control. The positional control of these valves usually presents a challenge, since they present nonlinearities as dead zones, hysteresis, and static and dynamic friction. Among these nonlinearities, we can mention the occurrence of the grip and slip (stick-slip) phenomenal on the valve stem due to static friction (stiction) between its moving parts. This phenomenon may compromise position control that causes a regime error and even generates periodic oscillations, also known as limit cycles, which force the process variables to exceed their limits of operation, safety, and efficiency, causing a premature degradation not only in the valve, but in all components of the control mesh [1] which results in losses in productivity. $30 \%$ of industrial processes present oscillations are caused by problems in control valves [2]. The average cost of maintaining a valve is between US\$ 400.00 and US\$ $2,000.00$ [3] and a production halt for maintenance routines may cause significant financial losses. Thus, the importance of the study and elaboration of viable solutions for the problems of grip in control valves are evident.

Stick-slip phenomenon needs to be represented by a mathematical model that approaches its physical characteristics. Moreover, accurate models that allow the identification and quantification of stiction are critical for their compensation by means of a model-based control strategy. However, a problem associated with its modeling and subsequent control resides in the fact that stiction presents stochastic characteristics [4]. Due to this stochastic nature, at each time, different control signal values are needed to overcome static friction in the same specific position, and different values of minimum speed are required so that the valve does not grip during opening or closing procedure, being not compatible with traditional model-based controllers. In addition to the problems inherent to the modeling and control of electropneumatic valves, the change of its parameters over time (caused by the wear of its mechanical components) makes the control and maintenance of production rates even more challenging, increasing the importance of periodic reidentification processes. These parameter changes can generate 
mismatch problems between the nominal model and the actual behavior of the plant, which may cause losses on the control loop efficiency, as well as nonfeasibility problems in the optimization process when a predictive controller is used.

PID controllers are the most used to control valves and also in a wide variety of industrial processes that include them, due to their robustness and satisfactory performance [5]. However, the inherent complexity of the stiction and other nonlinearities of control valves significantly reduce the performance of PID controllers, rendering them unable to drive the system to a desired position, which causes oscillations in the process control plant. These facts justify the rise of several approaches that treat stiction compensation and that preferably deal with stress constraint. Furthermore, other relevant characteristics in the performance of controllers, such as the control effort, may not be contemplated in cases where the control valves are gripped. An alternative is the adoption of Model-Based Predictive Controllers (MPC), since they contemplate these characteristics and their efficiency in the control of systems with Multiple Inputs and Multiple Outputs (MIMO), unlike the PID that is used in Single Input and Single Output (SISO).

MPC emerged in the late 70s $[6,7]$ and refer to a family of controllers that use the explicit process model to predict the future behavior of the dynamic system [8]. Initially designed and implemented in power plants and oil refineries, its use extends to several different areas in the present day. This can be partially justified by the difficulty of the classic controllers in dealing with the complexity, with operational constraints, MIMO systems, and nonlinearities of the current systems. These controllers use the system linear model to estimate the output trajectory $N$ steps ahead, into the future, as well as the control signal trajectory $M$ steps ahead, into the future. These signals are calculated by the minimization of an objective function defined in an optimization problem that may or may not consider the system constraints. The solution of the optimization problem can be obtained by linear programming, quadratic programming, min-max method, and multicriteria optimization [9]. MPC are heavily dependent on the type of model used to represent the dynamics of the process, which, in some cases, may be a disadvantage. Linear models are commonly described in the form of impulse response, step response, transfer function, and the state space model [10]. In MPC, state space models are most commonly used compared to others.

Although many industrial processes can be controlled by controllers based on linear models, there are processes that have many nonlinearities, which makes these controllers unsatisfactory. Therefore, the search for solutions based on nonlinear models has become attractive, such as neural controllers, fuzzy controllers, and predictive controllers based on nonlinear models (NMPC). This work proposes the use of NMPC, since it has a direct relation with the mathematical model that represents the system. On the other hand, the use of NMPC is a challenge due to the wide variety of nonlinearities present in physical systems. Some alternatives to deal with nonlinearities can be found in the literature, such as controllers based on the Hammerstein Model, Volterra Model, Wiener Model, and bilinear models. But for this, it is necessary to represent the system or process by one of these specific models, which becomes a limitation, because the nonlinearity in these models is static and stiction is stochastic parameter. Another alternative is the NMPC using successive linearization [13]. However, this approach requires considerable computational effort due to some computationally complex mathematical operations. In this paper, a nonlinear predictive controller based on a state space model structured in a similar to [13] was modified for the proposed compensation methodology in position controller system of the valve.

Kayihan and Doyle [14] proposed the use of an inputoutput linearization (IOL) scheme for friction compensation in pneumatic valves. However, it can only be applied to valves with an internal controller. Hägglund [15] describes a compensation technique for static friction in pneumatic valves, called Knocker. A similar proposal to Knocker was presented by Ivan and Lakshminarayanan [16], where the compensator received the denomination Constant Reinforcement (CR). These two methods use PID controllers and may not reach the above-mentioned operational constraint requirements, in addition to generating a control signal with reasonable variability and in some cases taking a long stabilization time.

This paper proposes the implementation of a nonlinear, iterative, predictive control system for stiction compensation. This system is able to lead the valve to different setpoints, which reduces the oscillations presented in the previous papers. For this, the basic principle of the proposed system is not to try to compensate stiction, but to use it as an advantage by designing a nonlinear predictive controller that incorporates minimum and maximum speed restrictions on the valve excursion; it decelerates and forces the valve to hold as close as the desired setpoint. In order to do this, a table that is updated with the excursion speed and control signal necessary to initiate slip phase was used. This update occurs every time the valve is grasped.

For better control dynamics, the concept of variable constraints was incorporated into the predictive controller, as well as the need to store the last ten (10) values in the stiction table to each setpoint aiming at calculating the medium value to compensate the stochastic character of the stiction [4]. Based on these parameters, the adopted predictive control strategy proved itself robust, capable of dealing with variations in valve parameters value, such as changes of $20 \%$ in the spring constant and the stiction value which causes mismatch between controller model and valve model.

This paper is organized as follows: Section 2 presents the linear displacement valve model and its electropneumatic control. In Section 3, a mathematical model of the friction force on the diaphragm-spring electropneumatic valves stem is presented. Section 4 presents brief explanation of the nonlinear predictive control based on the state space representation model. In Section 5, the proposed method is presented. In Section 6, simulation responses of the control methodology proposed are showed in five different situations. Finally, Section 7 draws conclusions and proposals for future work are suggested. 


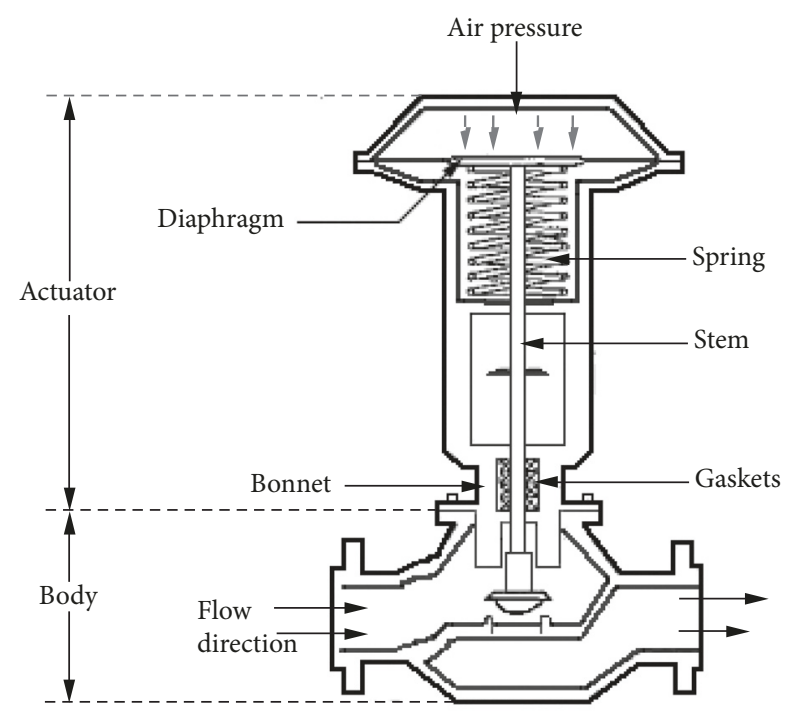

FIGURE 1: Body and actuator of a control valve with pneumatic actuator (adapted from [11]).

\section{Valves}

Valves are devices that are able to restrict or allow the passage of a fluid in response to a control signal. Valves can be classified as manual, self-regulatory, and controlled, which use an auxiliary force to operate, and its operation is driven by a control signal. This auxiliary force comes through the combined action of the actuator and the positioner. Similarly, there are several types of actuators and positioners. The types of actuators include the handwheel, manual lever, electric motor, pneumatic, solenoid, hydraulic piston, and self-actuation, where more than $90 \%$ of these are pneumatic actuators [15]. They all have a particular composition due to their type. This paper focuses on electropneumatic linear displacement control valves which consist basically of two main sets: the actuator and the body. The actuator, in this case, converts the electrical signal into a corresponding pressure to move valve stem. The conversion is done through a linear relationship between current and pressure, which, in turn, uses air pressure on one or both sides of a diaphragm to provide the force to position the valve.

The body assembly is basically divided into the following subsets: body, internal components, bonnet, and lower flange. In some valve types, the body and bonnet form one set simply called the body. In other valve types, there is no lower flange; hence, it makes the whole valve body the part that comes into direct contact with the fluid. The valve types are classified according to their body types. These valves are divided into the following:

(I) Linear displacement: conventional globe, three-way globe, cage globe, angle globe, diaphragm, and guillotine.

(II) Rotary displacement: butterfly, ball, shutter eccentric, and sphere segment.

One of the key elements of a valve is the box set of gaskets that serves to seal against process fluid leakage. Figure 1

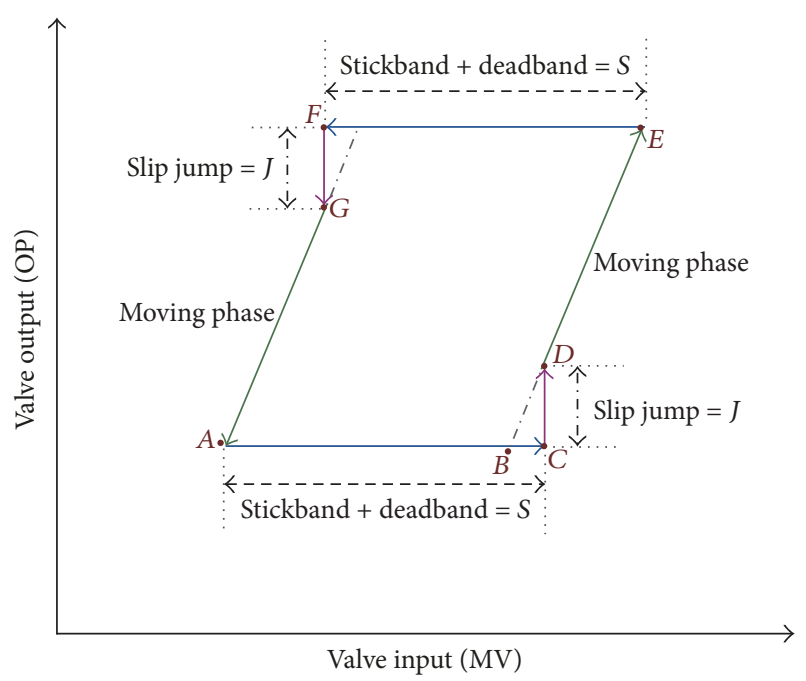

FIGURE 2: Standard signature curve of a valve with stiction (adapted from [12]).

represents the body and actuator of a linear displacement control valve (globe type).

Two problems related to the difference between the atmospheric and internal valve pressures may arise in cases when the gaskets box is not correctly sealed. If internal pressure is higher, it can cause leakage from the valve's internal fluid to the environment. In those cases where these valves are used in flammable or toxical fluid pipes, the gaskets must be firmly pressed in order to prevent any leakage. This may cause an increase in stiction and consequently the problems associated with this phenomenon.

The desired characteristics of the material used in the composition of gaskets are elasticity, low coefficient of friction, pressure, temperature, and resistance to corrosion caused by the process fluid. The most common materials used in the construction of gaskets are Teflon and impregnated asbestos.

Stiction is the most common type of nonlinearity in electropneumatic control valves [1]. The sealing rings (also known as gaskets) are the principal responsible component in the valve for the phenomenon.

The stiction phenomenon can be better explained by the valve input-output behavior, as illustrated in Figure 2. In the absence of stiction, the valve moves along the dotted line trace that passes through the origin. Any controller output signal (OP) would result in the same change in the value of the manipulated variable (MV). However, for a stuck valve, static and dynamic friction components must be taken into account. The input-output can therefore be described by four components: deadband, stiction band, slip jump $(J)$, and mobile phase.

Let us assume that in point $A$, Figure 2, the valve stem is stopped. Shortly thereafter there was a reversal in the movement direction where $\mathrm{MV}$ is constant, as long as the valve is stuck by the action of static friction force $\left(F_{s}\right)$. The deadband range $(A B)$ exists due to the presence of Coulomb friction $\left(F_{c}\right)$ (a constant force that operates in the opposite 
direction of the movement). Point $B C$ corresponds to the band stiction $(J)$, where as in point $C$ the $\mathrm{MV}$ value is abruptly changed (stiction jump- $J$ ), due to the applied force and consequently valve stem sliding. After that, MV varies progressively (movement phase); it is only opposed by the dynamic frictional force $\left(F_{v}\right)$. When the speed of the valve stem is too low, it grasps again and the movement inversion does not occur. The band formed by the deadband and the stiction band will be referred to as $S$. In an attempt to overcome the gripping, the actuator may jump around the desired position that causes the temporary oscillatory behavior of the manipulated variable. This behavior of the manipulated variable is also propagated to the process variable which, in turn, is how the stiction disturbance contributes to the instability of the process and, consequently, to the loss of performance of the control loop.

As discussed above, friction is a force that directly influences the valve dynamics. Its modeling is fundamental, in order to detect and quantify the stiction force to controller design that focuses on its compensation.

\section{Modeling}

Several models have been proposed to represent stiction dynamics in pneumatic valves using phenomenological approaches. Reference [17] presents several of these models. Since then, new models have been created using the inputoutput relationship. References [12, 18-20] presented new models, where [18] presents one parameter based model, and the other works are based on two-parameter model.

3.1. Valve Model and Friction Force Model. Eight friction models were selected by [2] (physical and based on process operating data models) in order to conduct a performance comparison among them: [21], the seven-parameter models of [22], Siskin model [23], the one parameter model of [18], [12] model, [19] model, and [20] model. In conclusion, the best representations of friction in a control valve are Karnopp, Siskin, and Kano models, since they had been approved in all tests.

In this paper, the physical friction model of Karnopp was selected, since it adequately represents the phenomenon of stiction, as concluded in the work of [2], and it models friction as a static function of speed. In this way, the position and speed of the stem can be manipulated directly by the predictive controller, which is the basis of the methodology proposed by this paper.

In order to obtain the valve model, a force balance is applied to the valve stem according to Newton's second law. It is assumed that the input signal is the signal defined by the controller, converted to pressure measurement unit, and the system output is the valve stem position (usually normalized between 0 and $100 \%$ ), as shown in

$$
\begin{aligned}
m \ddot{x}(t)= & F_{\text {pressure }}-F_{\text {spring }}-F_{\text {friction }}-F_{\text {fluid }} \\
& -F_{\text {settlement }},
\end{aligned}
$$

where

(i) $m$ is the mobile mass parts (obturator + stem); (ii) the position of valve is $x(t)$;

(iii) $F_{\text {pressure }}=S_{a} P$ is the force applied by the pressure on the diaphragm actuator; $S_{a}$ is the diaphragm area and $P$ is the pressure on the actuator;

(iv) $F_{\text {spring }}=K_{m} x(t)$ is the force applied by the actuator spring and $K_{m}$ is the spring elastic constant;

(v) $F_{\text {fluid }}$ is the force applied by the process fluid in the valve obturator;

(vi) $F_{\text {friction }}$ is the total friction force on stem;

(vii) $F_{\text {settlement }}$ is a settlement external force (necessary to lock the valve on the seat) of the shutter in the seat of the valve; it only acts on the end of the stem excursion.

Reference [14] assumed that the force of the fluid $F_{\text {fluid }}$ can be neglected, since it is very small compared to the other forces involved. But [14] does not present results to prove that $F_{\text {fluid }}$ can, in fact, be neglected. Reference [24] conducted laboratory experiments to confirm such hypothesis. The settlement force $F_{\text {settlement }}$ also will be neglected since it acts only in the end of the valve actuation range, when there is no further stem movement made; only the shutter is being forced against the seat.

Therefore, (1) can be written as

$$
m \ddot{x}(t)=F_{\text {pressure }}-F_{\text {spring }}-F_{\text {friction }} .
$$

The main difference between the most commonly used physical models for control valve lies in the way that the friction force is calculated. Therefore, in [2], a study for the application of different friction models to a control valve is conducted. There are more complex models in the literature as described in [22] using seven parameters. These complex models, in general, have parameters in their model of difficult access. Thus, it is important to select a model that presents simplicity and, at the same time, adequately describes the friction.

In this paper, the Karnopp model in [21] is adopted, since it easily deals with the null speed detection problem, an important point in valve modeling, through the definition of a minimum speed $D V$. The model used is represented by equations below:

$$
\begin{aligned}
& F_{\text {friction }} \\
& = \begin{cases}F_{\text {friction }}(\dot{x}(t)), & \text { if }|\dot{x}(t)| \geq D V, \\
F_{e}, & \text { if }|\dot{x}(t)|<D V,\left|F_{e}\right| \leq F_{s}, \\
F_{s} \cdot \operatorname{sign}\left(F_{e}\right), & \text { if }|\dot{x}(t)|<D V,\left|F_{e}\right|>F_{s} .\end{cases} \\
& F_{\text {friction }} \text { is the total friction force defined as } \\
& F_{\text {friction }}(\dot{x}(t))=\gamma \operatorname{sign}(\dot{x}(t))+F_{v} \dot{x}(t), \\
& \gamma=\left[F_{c}+\left(F_{s}-F_{c}\right) e^{\left(\dot{x}(t) / V_{s}\right)^{2}}\right],
\end{aligned}
$$

where

$$
\operatorname{sign}(\dot{x}(t)) \begin{cases}1, & \text { if } \dot{x}(t)>0, \\ 0, & \text { if } \dot{x}(t)=0, \\ -1, & \text { if } \dot{x}(t)<0 .\end{cases}
$$




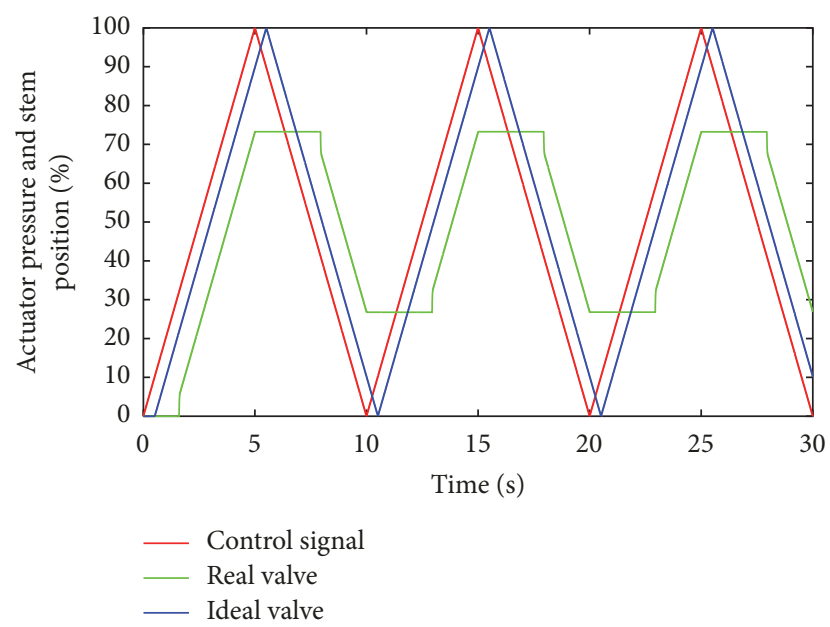

FIGURE 3: Valve responses to an input of a triangular signal.

TABLE 1: Nomenclatures in mathematical Karnopp model.

\begin{tabular}{lcc}
\hline Parameter & SI base units & Description \\
\hline$F_{e}$ & $\mathrm{~N}$ & The sum of the external forces: \\
$F_{c}$ & $\mathrm{~N}$ & $F_{\text {pressure }}-F_{\text {spring }}$ \\
$F_{s}$ & $\mathrm{~N}$ & Coulomb friction coefficient \\
$F_{v}$ & $\mathrm{~N}$ & Static friction coefficient \\
$V_{s}$ & $\mathrm{~m} / \mathrm{s}$ & Viscous friction coefficient \\
$x(t)$ & $\mathrm{m}$ & Stribeck speed \\
$\dot{x}(t)$ & $\mathrm{m} / \mathrm{s}$ & Stem position \\
\hline
\end{tabular}

A presence of static friction (stiction) causes a reduced control valve working range. In many cases, the valve stem signals are normalized to a corresponding value between the MV limit ranges. This way, when the friction is absent, the stem position curve behaves as a replica of the controller signal curve, which can be observed in the red and blue color curves, respectively. However, in the green color curve, in Figure 3, the actual behavior of the stem is observed, where it presents a smaller amplitude than the blue curve, due to the phenomenon of the stick-slip caused by the presence of the friction. In Figure 3, the triangular input signal and the outputs are displayed as time works for a valve which is considered in perfect condition and, in the case of the green curve, the friction action is present. The application of a triangular signal to the valve is a commonly used method when defining the valve signature curve.

Table 1 describes the nomenclature to be adopted in the Karnopp model equation and its respective units of measure.

As previously mentioned, valves are elements that present many nonlinearities and a stochastic parameter (stiction). Beyond that, the parameters values of the valve can suffer variations causing mismatching between the dynamic system and the phenomenological model.

One of the parameters that suffers a great degradation due to the oscillations caused by the stiction phenomenon is the spring constant, which tends to decrease when compared to new valves. This forces a periodic reidentification process of the model parameters and consequently controller parameters recalculation.

Valves that operate in external environments or with fluids that suffer changes with temperature may represent a challenge, since these temperature changes affect the spring elastic constant and the friction in the gaskets, due to thermal expansion.

The use of a PID controller forces the system into an oscillatory cycle. This is caused by the integrative action component of the controller when the valve grasps in a wrong position. In this situation, the integrative action keeps increasing the control input value until the valve starts moving. However, when this happens, the valve usually slips, and its position jumps over the reference, completing the cycle.

3.2. Valve Model in the State Space Representation. From the total friction force model described by (4) and from equation of the valve model, we have

$$
\begin{aligned}
m \ddot{x}(t)= & F_{\text {pressure }}-F_{\text {spring }}-F_{v} \dot{x}(t) \\
& -\left[F_{c}+\left(F_{s}-F_{c}\right) e^{\left(\dot{x}(t) / V_{s}\right)^{2}}\right] \operatorname{sign}(\dot{x}(t)),
\end{aligned}
$$

where forces $F_{\text {pressure }}$ and $F_{\text {spring }}$ are, respectively, defined by

$$
\begin{aligned}
F_{\text {pressure }} & =S_{a} P, \\
F_{\text {spring }} & =K_{m} x(t),
\end{aligned}
$$

where $S_{a}$ is the diaphragm area, $P$ is the pressure in diaphragm, and $K_{m}$ is the elastic constant of the spring. From (7) in (6)

$$
\begin{aligned}
m \ddot{x}(t)= & S_{a} P-K_{m} x(t)-F_{v} \dot{x}(t) \\
& -\left[F_{c}+\left(F_{s}-F_{c}\right) e^{\left(\dot{x}(t) / V_{s}\right)^{2}}\right] \operatorname{sign}(\dot{x}(t)) .
\end{aligned}
$$

Parameters inherent to the actuator such as maximum pressure $\left(P_{\max }\right)$, minimum pressure $\left(P_{\min }\right)$, maximum current $\left(I_{\max }\right)$, and minimum current $\left(I_{\min }\right)$ are used so that a linear relationship can be established in the pressure conversion in the diaphragm of valve $P$, as a function of the current signal $u(t)$ in $\mathrm{mA}$. The dynamics in a linear converter can be neglected because it has a faster dynamic in relation to the system.

$$
\begin{aligned}
K_{p} & =\frac{\Delta P}{\Delta I}=\frac{P_{\max }-P_{\min }}{I_{\max }-I_{\min }}, \\
P & =K_{p} u(t) .
\end{aligned}
$$

From the substitution of (10) in (8), we reach

$$
\begin{aligned}
m \ddot{x}(t)= & S_{a} K_{p} u(t)-K_{m} x(t)-F_{v} \dot{x}(t) \\
& -\left[F_{c}+\left(F_{s}-F_{c}\right) e^{\left(\dot{x}(t) / V_{s}\right)^{2}}\right] \operatorname{sign}(\dot{x}(t)) .
\end{aligned}
$$

Finally, in order to define the position and the speed of the stem as state variables, as well as by algebraically 
manipulating equation (11), it gives a state space model representation, based only on the total frictional force, that is, the classic model found in the literature to represent the frictional force.

$$
\begin{aligned}
x_{1}(t) & =x(t), \\
x_{2}(t) & =\dot{x}(t) \\
& \Downarrow \\
\dot{x}_{1}(t) & =\dot{x}(t), \\
\dot{x}_{2}(t) & =\ddot{x}(t), \\
\dot{X}(t) & =A X(t)+B u(t)+K_{n L} \operatorname{sign}\left(x_{2}(t)\right), \\
y(t) & =C X(t),
\end{aligned}
$$

where $A, B, C, K_{n L}$, and $X(t)$ are matrices defined by

$$
\begin{aligned}
X(t) & =\left[\begin{array}{l}
x_{1}(t) \\
x_{2}(t)
\end{array}\right], \\
\dot{X}(t) & =\left[\begin{array}{l}
\dot{x}_{1}(t) \\
\dot{x}_{2}(t)
\end{array}\right], \\
A & =\left[\begin{array}{cc}
0 & 1 \\
-\frac{K_{m}}{m} & -\frac{F_{v}}{m}
\end{array}\right], \\
B & =\left[\begin{array}{c}
0 \\
S_{a} K_{p} \\
m
\end{array}\right], \\
C & =\left[\begin{array}{ll}
0 & 1
\end{array}\right], \\
K_{n L} & =\left[\begin{array}{c}
1 \\
-\frac{1}{m}\left[F_{c}+\left(F_{s}-F_{c}\right) e^{\left(x_{2} / V_{s}\right)^{2}}\right.
\end{array}\right] .
\end{aligned}
$$

The adoption of the classical friction force model in place of the Karnopp model in the state space representation is due to the fact that some model-based controllers, such as predictive controllers, require the model to be represented in state space, which is difficult to obtain from the Karnopp model. In addition, this representation also needs to be in discrete time that implies the use of discretization techniques for its practical application in the control of electropneumatic valves.

\section{Nonlinear MPC-NMPC}

Systems that can be represented in the following manner are considered in this work:

$$
\begin{aligned}
x(k+1)= & f(x(k), u(k)) \cdot x(k)+g(x(k), u(k)) \\
& \cdot u(k) \\
y(k)= & C x(k),
\end{aligned}
$$

where $x \in R^{n}$ is the state vector, $u \in R^{m}$ is the input vector, $y \in$ $R^{P}$ is the output vector, and $k \in \mathbb{N}$ is the sampling time. It is assumed that the controlled system and state space variables are subject to the following linear constraints:

$$
\begin{aligned}
& G x(k) \leq \rho, \\
& H u(k) \leq \mu .
\end{aligned}
$$

The predictive control scheme defines, at each time step $k$, an input trajectory $\widehat{u}(k), k=0,1, \ldots, H_{u}$, that satisfies the following nonlinear optimization problem:

$$
\begin{aligned}
& \min _{\widehat{x}(k+i), \widehat{u}(k+j)} J(k) \\
& =\sum_{i=1}^{H_{p}}\|r(k+i)-C \widehat{x}(k+i)\|_{Q} \\
& +\sum_{H_{u}-1}^{j=0}\|\widehat{u}(k+j)\|_{R}, \\
& \text { Subj. to } \hat{x}(k+1+i) \\
& =f(\widehat{x}(k+i), \widehat{u}(k+i)) \hat{x}(k+i) \\
& +g(\widehat{x}(k+i), \widehat{u}(k+i)) \widehat{u}(k+i), \\
& \hat{x}(k)=x(k), \\
& \widehat{u}(k+j+1)=\widehat{u}(k+j), \\
& G \hat{x}(k+i) \leq \rho, \\
& H \widehat{u}(k+i) \leq \mu,
\end{aligned}
$$

where the objective function $J(k)$ is the performance index of the finite prediction horizon; $r(k+i)$ is the future reference trajectory to be followed by the output $y(k)=C x(k) ; H_{p}$ is the prediction horizon, it is constant, and $H_{p} \in \mathbb{N} ; H_{u}$ is the control horizon, it is constant, and $H_{u} \in \mathbb{N} ; Q$ is the tracking error weighting matrix; $R$ is the control effort weighting matrix (being $Q$ and $R$ positive definite); $\widehat{x}$ is the state vector predicted by the model in (15), while in (17) $\widehat{x}(k)=x(k)$; and $\widehat{u}$ is the input vector to be determined in a manner that optimizes the performance index.

The $J(k)$ minimization results in the control sequence $\widehat{u}(k+j)$ that optimizes the predicted behavior of the system in the prediction horizon $H_{p}$. The mathematical model does not represent with perfect accuracy the behavior of real systems, the occurrence of disturbances, and the presence of noise, among other difficulties that arise in practical applications, and the predictive control strategy employs the movable horizon principle that consists in the application of the first element $\widehat{u}(k \mid k)$ of the control sequence computed and the subsequent recalculation of the optimization problem in the next time step.

Prior knowledge of the full state vector $x(t)$ is required for the solution of the optimization problem which characterizes a full state feedback structure.

The optimization (17) is a nonlinear, nonconvex problem and may depend on many variables that depend on the 
system dimension and the length of the horizons $H_{p}$ and $H_{u}$. The solution for this problem presents a considerably high computational cost, hindering its application on systems with fast dynamics, where the sampling time is not high enough so that the problem solution can be computed in a feasible time period. However, if the system dynamic is linear, explicit linear equations can be defined in a way that represents the predicted states $\widehat{x}(k+i)$ as functions of the inputs variables $\widehat{u}(k+j)$. This way, the number of variables is greatly reduced and the problem can be formulated as a quadratic optimization problem, enabling the usage of efficient and fast algorithms. Thus, the methods that have been proposed for the resolution of nonlinear predictive control problems can be roughly divided into two categories: the first one proposes fast algorithms to solve the problem (17) and the second one proposes different ways to linearize the model (15), so that efficient methods for quadratic optimization can be employed. The method employed in this work fits the second category and will be described in the following section.

4.1. Iterative Compensation Algorithm. The one-step state prediction is considered in the following equation:

$$
\begin{aligned}
\widehat{x}(k+1+i)= & f(\widehat{x}(k+i), \widehat{u}(k+i)) \hat{x}(k+i) \\
& +g(\widehat{x}(k+i), \widehat{u}(k+i)) \widehat{u}(k+i) .
\end{aligned}
$$

It is important to notice that if the values of $\widehat{x}(k+i)$ and $\widehat{u}(k+i)$ were numerically defined through the prediction horizon $i=$ $0, \ldots, H_{p}-1$, the following time-variant linear state equation would be defined as

$$
\widehat{x}(k+1+i)=\widetilde{A}_{i}(\widehat{x}, \widehat{u}) \widehat{x}(k+i)+\widetilde{B}_{i}(\widehat{x}, \widehat{u}) \widehat{u}(k+i) .
$$

Application of the movable horizon principle in (19) is

$$
\begin{aligned}
\widehat{x}(k+1+i \mid k) \\
=\widetilde{A}_{i}(\widehat{x}(k+i \mid k), \widehat{u}(k+i \mid k)) \widehat{x}(k+i \mid k) \\
\quad+\widetilde{B}_{i}(\widehat{x}(k+i \mid k), \widehat{u}(k+i \mid k)) \widehat{u}(k+i \mid k),
\end{aligned}
$$

where $\widetilde{A}_{i}(\widehat{x}, \widehat{u})=f(\widehat{x}(k+i), \widehat{u}(k+i))$ and $\widetilde{B}_{i}(\widehat{x}(k+i \mid k), \widehat{u}(k+i \mid$ $k))=g(\widehat{x}(k+i), \widehat{u}(k+i))$. However, $\widehat{x}(k+i)$ and $\widehat{u}(k+i)$ values are not known throughout the horizon, since the values $\widehat{x}(k+$ $i)$ depend on the future values $\widehat{u}(k+i)$. Nevertheless, in [13], an algorithm that promotes the convergence of states to the correct predicted values is described and defined as follows.

For each sample time $k$, one has the following.

Step 1. Set up the input vector $U(k)=U^{*}(k)$.

Step 2. It is considered that $x(k)$ is measured in the plant; it applies $U(k)$ to the model (19) to obtain the prediction vector states $\widehat{X}(k)=\left[\widehat{x}(k+1 \mid k), \widehat{x}(k+2 \mid k), \ldots, \widehat{x}\left(k+H_{p}-1 \mid\right.\right.$ $\left.k), \widehat{x}\left(k+H_{p} \mid k\right)\right]$ over the prediction horizon of $H_{p}$.

Step 3. Apply $U(k)$ and $\widehat{X}(k)$ to model state prediction one step ahead as in (20). In order to obtain linear state equations over $H_{p}$ prediction horizon from current time $k, \widehat{x}(k+1+i \mid$
$k)=A(k+i \mid k) \hat{x}\left(k_{i} \mid k\right)+B(k+i \mid k) \widehat{u}(k+i \mid k)$. Note that $\widetilde{A}(\widehat{x}(k+i \mid k), \widehat{u}(k+i \mid k))$ and $\widetilde{B}(\widehat{x}(k+i \mid k), \widehat{u}(k+i \mid$ $k))$ are functions of the states and inputs along the prediction horizon, while $A_{i}(k+i \mid k)$ and $B_{i}(k+i \mid k)$ are matrices numerically defined at the end of Step 3 .

Step 4. From the linear model generated in Step 3, define the quadratic optimization problem based on (17) (problem equation optimization with constraints), to meet a new vector prediction of entries, that is, an optimal control signal vector $U^{*}(k)$.

Step 5. If it is confirmed that the values of $U(k)$ and $U^{*}(k)$ follow a certain criteria convergence, it is assumed that the algorithm arrived at its end that allows the implementation of the first value $U(k)$ and moving the plant to the next instant $k$ sampling. Otherwise, it returns to Step 1.

If this algorithm converges, then, the values $\widehat{x}(k+i)$ and $\widehat{u}(k+i)$ predicted by the time-variant linear model would coincide with the ones predicted by the original now linear model. Thus, if the optimization problem has a feasible solution, so there is a guarantee that the constraints (16) will be respected throughout the horizon prediction. This fact represents an advantage over methods based on linearized models, since, as close as these models may represent the original model, they cannot guarantee constraints satisfaction.

\section{Proposed Method}

The proposed method is based on two steps that continuously occur during the operation of the valve. The first step is responsible for recording, on a table, the valve speed whenever a stop is detected, as well as the control action determined by the controller an instant before a slip occurs. The second process updates the quadratic programming constraints considering the information registered in the first step.

5.1. Learning Process. It consists of the assembly and update of stiction velocities table. Every moment a valve stop is detected, the algorithm records the position and speed of the valve stem (at the moment before your speed becomes null) in the table.

5.2. Stiction Treatment Process. At each instant, the algorithm checks if there is a register that relates the stem speed to the desired valve position. If one or more of these registers exist in the table, we use the mean of these speeds in a mathematical equation to move the speed constraint of the NMPC. As a consequence, the optimization problem calculates a control trajectory that causes the stem to move with the speed required to adhere as close to the desired valve position as possible. On the other hand, if there is no register in the table that relates speed to a valve position, it will be immediately registered in the table. We will refer to this movable constraint of speed as restVel. The absolute error between a current position of the stem and the setpoint of the position control system is another parameter that can 
TABLE 2: Stiction table in instant $k$.

\begin{tabular}{lcccc}
\hline Pos $(\mathrm{cm})$ & $x_{2}\left(k_{1}\right)$ & $x_{2}\left(k_{2}\right)$ & $x_{2}\left(k_{3}\right)$ & Average speed $(\mathrm{cm} / \mathrm{s})$ \\
\hline 15 & 0.01 & 0.03 & 0.02 & 0.02 \\
10 & Null & Null & Null & Null \\
5 & 0.05 & 0.04 & 0.03 & 0.04 \\
\hline
\end{tabular}

be considered in the equation of the movable constraint of speed. The valve speed constraint must not be too strict when the absolute difference between the controller and the valve is considerable, as this may lead to an infeasible optimization problem. A possible approach for mathematical equation of the movable constraint of speed as a function of the absolute error may be as explained below:

$$
\text { restVel }=K \cdot \mid \text { error } \mid+v_{\mathrm{Ag}},
$$

where $v_{\mathrm{Ag}}$ is the value registered in the table for the speed the valve had before sticking, error is the difference between the current valve position and the controller reference, and $K$ is the weight constant. Note that the lower the value of the variable $K$ is, the faster the value of the speed constraint will reach the value registered in the table for the speed constraint.

In the tests phase, the formulation to update restVel caused some feasibility problems to the solution of the optimization problem for large values of $K$. The following formulation is assumed, which updates the values in a smoother manner:

$$
\text { restVel }=\alpha \cdot\left(-e^{-\beta \cdot \mid \text { error } \mid}+1\right)+v_{\mathrm{Ag}},
$$

where $\alpha$ and $\beta$ are two tuning parameters and $e$ is the exponential function, $\alpha$ must have a greater value than the estimated maximum speed value for the valve, and the value of $\beta$ is an arbitrary value that should be large enough so that the valve sticks near the reference.

From the calculated value of restVel, the value of the speed constraints $\theta$ is set as

$$
-V_{\min } \leq \theta \leq \text { restVel, }
$$

if the valve position value is smaller than the reference value. Otherwise, the speed constraint is defined as

$$
\text { -restVel } \leq \theta \leq V_{\text {max }},
$$

where $V_{\min }$ and $V_{\max }$ are estimates of minimum and maximum valve speed, respectively.

Whenever there is a need to change the position of the valve, the controller will increase the absolute value of the control action until it reaches the minimum necessary force to detach the valve. So, whenever a slip occurs, the control action $\Gamma$ applied at the moment to the valve is stored in the table. This information is used to accelerate the detachment of the valve whenever it is stuck again in the same position registered in the table. It is considered that the control action varies slowly until it reaches the value of $\Gamma$, and the control action $u$ may be limited by the following restriction,

$$
\Gamma \leq u
$$

in case the controller reference is greater than the actual position. Otherwise,

$$
u \leq \Gamma
$$

5.3. Example. As an example, a controller with a reference set to 10 (ten) $\mathrm{cm}$ in initial instant $k$ has the configuration shown in Table 2.

For the determined reference, there is no particular registered value for the stiction speed in the table. Thus, the controller does not consider restrictions on the speed variable, forcing the valve into an oscillatory cycle around the reference.

From the values recorded in the table for the case where the reference is set to 5 (five) $\mathrm{cm}$ and $v_{\mathrm{Ag}}=0.04 \mathrm{~cm} / \mathrm{s}$, the algorithm will calculate a new value for the speed constraint in the optimization problem (17). With the $v_{\mathrm{Ag}}$ value calculated, setting values for $\alpha=5, \beta=150$, using (22),

$$
\text { restVel }=5 \cdot\left(-e^{-150 \cdot|1-0.03|}+1\right)+0.04=5.04
$$

Since the valve position value is lower than the reference value, the new speed constraint $\theta$ is set as

$$
-V_{\min } \leq \theta \leq 5.04
$$

Thus, the algorithm works with variable constraints on the valve speed variable that increases their strictness as the valve approaches the control reference. In this manner, the stiction compensation scheme aims to force the valve to stop as close as possible to the controller reference, cancelling the oscillatory movement around the setpoint that characterizes the stiction disturbance. The control action values necessary to start a valve movement whenever it is stuck can also be stored in the table. The control action values that initiate valve movement must also be stored in the table whenever it gets stuck. These values are used to update the constraints on the input variable which speeds the valve detachment process.

\section{Tests and Results}

For simulation purposes, the valve and controller models, as well as the control algorithms and the proposed system, were implemented in the software Matlab ${ }^{\circledast / S i m u l i n k . ~ T h e ~}$ Karnopp model was used to represent the friction force in the valve model simulation while the classic model was employed in the NMPC. In the discretization of the state space model (13), the differential equations were approximated by finite difference equations, $d x / d t \approx\left(x\left(t+T_{s}\right)-x(t)\right) / T_{s}$, and were 
TABLE 3: Parameters estimated valve control (adapted from [2]).

\begin{tabular}{lcc}
\hline Parameter & Value & Measurement unit \\
\hline$m$ & 1.361 & $\mathrm{Kg}$ \\
$S_{a}$ & 0.06452 & $\mathrm{~m}^{2}$ \\
$F_{s}$ & 1707.7 & $\mathrm{~N}$ \\
$F_{c}$ & 1423 & $\mathrm{~N}$ \\
$F_{y}$ & 612.9 & $\mathrm{~N} \cdot \mathrm{s} / \mathrm{m}$ \\
$K_{m}$ & 52538 & $\mathrm{~N} / \mathrm{m}$ \\
$V_{s}$ & 0.000254 & $\mathrm{~m} / \mathrm{s}$ \\
$D V$ & 0.0001524 & $\mathrm{~m} / \mathrm{s}$ \\
$K_{p}$ & $(0.75 \times 6894.757)$ & $\mathrm{Pa} / \mathrm{mA}$ \\
\hline
\end{tabular}

used with sampling time $T_{s}$. The result of the discretization can be checked in the equation below:

$$
\begin{aligned}
X(k+1) & =\bar{A} X(k)+\bar{B} u(k)+\bar{K}_{n L} \operatorname{sign}\left(x_{2}(k)\right), \\
y(k) & =C X(k),
\end{aligned}
$$

where $\bar{A}, \bar{B}, C, \bar{K}_{n L}$, and $X(k)$ are matrices defined by

$$
\begin{aligned}
X(k) & =\left[\begin{array}{l}
x_{1}(k) \\
x_{2}(k)
\end{array}\right], \\
C & =\left[\begin{array}{ll}
0 & 1
\end{array}\right], \\
\bar{A} & =\left[\begin{array}{cc}
1 & T_{s} \\
-\frac{K_{m}}{m} T_{s} & 1-\frac{F_{v}}{m} T_{s}
\end{array}\right], \\
\bar{B} & =\left[\begin{array}{c}
0 \\
\frac{S_{a} K_{p}}{m} T_{s}
\end{array}\right], \\
\bar{K}_{n L} & =\left[\begin{array}{c}
0 \\
-\frac{1}{m} T_{s}\left[F_{c}+\left(F_{s}-F_{c}\right) e^{\left(x_{2}(k) / V_{s}\right)^{2}}\right.
\end{array}\right] .
\end{aligned}
$$

In the linearization phase, it is necessary that (29) be in the format described by (15). For this, the matrix $\bar{K}_{n L}$ had to be incorporated on transition matrix of states $\bar{A}$ which implies that the initial course of the stem is obligatorily different from zero, $x_{1}(k) \neq 0$, and the discrete model (29) used in NMPC will be described by

$$
\begin{aligned}
& X(k+1)=\bar{\Phi} X(k)+\bar{B} u(k), \\
& y(k)=C X(k), \\
& \bar{\Phi}=\left[\begin{array}{cc}
1 & T_{s} \\
-\frac{\bar{\gamma} \cdot \operatorname{sign}\left(x_{2}(k)\right)+K_{m} x_{1}(k)}{x_{1}(k) m} T_{s} & 1-\frac{F_{v}}{m} T_{s}
\end{array}\right], \\
& \bar{\gamma}=\left[F_{c}+\left(F_{s}-F_{c}\right) e^{\left(\dot{x}(k) / V_{s}\right)^{2}}\right] .
\end{aligned}
$$

The numerical values of the parameters belonging to the valve model adopted in the simulations are shown in
TABLE 4: Nomenclatures in mathematics of the control strategy.

\begin{tabular}{lc}
\hline Parameter & Description \\
\hline$p$ & Number of outputs \\
$m$ & Number of inputs \\
$Q$ & Error weighting matrix \\
$R$ & Control action weighting matrix \\
$H_{p}$ & Prediction horizon \\
$H_{u}$ & Control horizon \\
$\alpha$ & Tuning parameter \\
$\beta$ & Tuning parameter \\
eye $(\cdot)$ & Identity matrix function \\
\hline
\end{tabular}

TABle 5: Predictive controller parameters.

\begin{tabular}{lc}
\hline Parameter & Value \\
\hline$H_{p}$ & 4 \\
$H_{u}$ & 4 \\
$Q$ & $\operatorname{eye}\left(p\left(H_{p}+1\right) \times p\left(H_{p}+1\right)\right)$ \\
$R$ & $10^{-5} I_{m} \cdot H_{u} \times m \cdot H_{u}$ \\
$T_{s}$ (sample time) & $10^{-4}$ seconds \\
$\alpha$ & 5 \\
$\beta$ & 150 \\
\hline
\end{tabular}

Table 3. Table 4 describes the nomenclature of control strategy adopted, as well as its respective dimensions. Similarly, in Table 5, there is information regarding the tuned predictive controller, where its restrictions are the state variable $x_{2}(k)$, the stem speed, and the control signal $u(k)$. The values of the $\alpha, \beta, H_{p}$, and $H_{u}$ tuning parameters of Table 5 were obtained empirically. In order to represent the existing measurement noise in industrial environments, a random signal with a mean power of $2 \%$ in relation to the measured signal was incorporated in all simulations in the measurement of the position variable.

For the correct operation of the system, the controller must be aggressive enough so that the valve does not stick too far from the desired reference. A more aggressive controller, along with minimum constraint on the input $u$, will also help to detach the valve when a change of reference is required.

In the following section, 5 simulations results will be presented. These simulations aim to explain the effectiveness of the proposed stiction compensation system when applied under different conditions. The trajectories of the stem position, the control signal, and the speed variable are illustrated.

6.1. NMPC Nonlinear in the Absence of Stiction $\left(F_{s}=0 \mathrm{~N}, F_{c}=\right.$ $\left.0 \mathrm{~N}, F_{v}=612.9 \mathrm{~N}\right)$. In Figures 4(a), 5(a), 6(a), 7(a), and 8(a), the blue line represents the position of the stem and the red line represents desired position, that is, the valve setpoint.

In the figures, it is possible to observe the absence of friction, and the predictive controller drives the stem position to the correct reference and, due to the aggressive tuning, causes an overshoot and oscillations during the transient system response. The decision to maintain the viscous friction parameter in the model during the simulation is explainable 


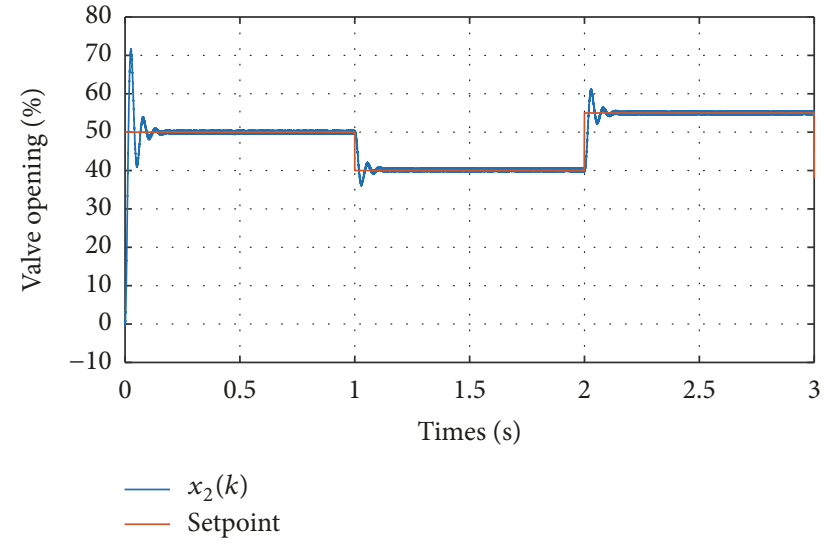

(a) Stem position chart

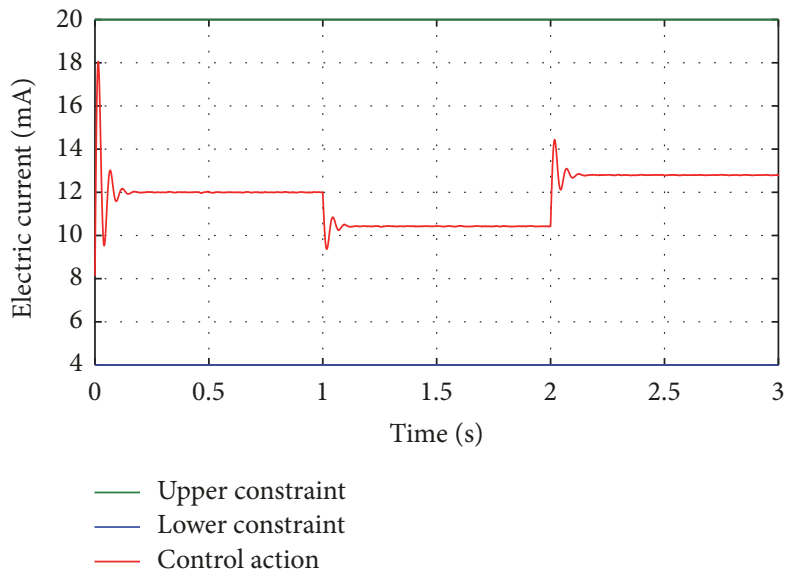

(b) Control signal chart

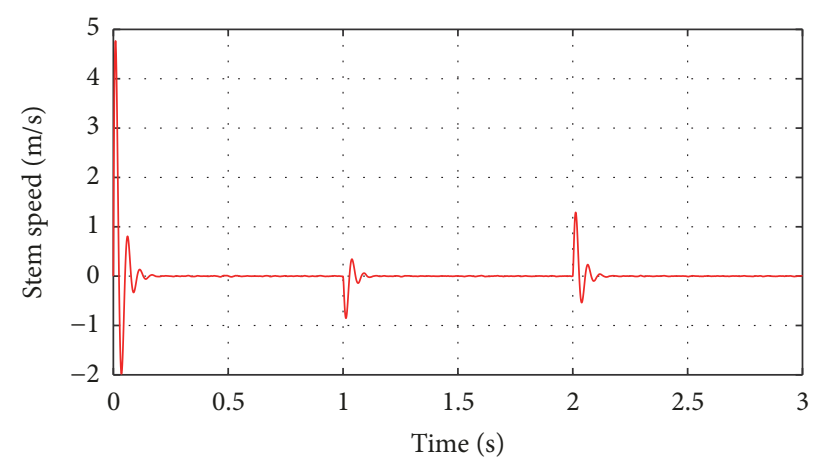

Upper constraint
_ Lower constraint
Stem speed

(c) Speed chart

Figure 4: Simulation 1.

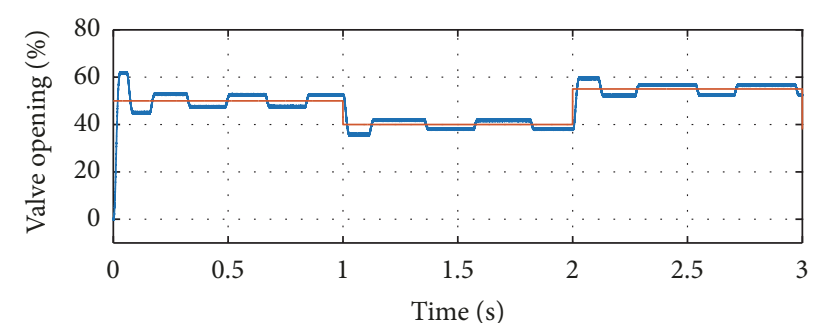

$x_{2}(k)$

Setpoint

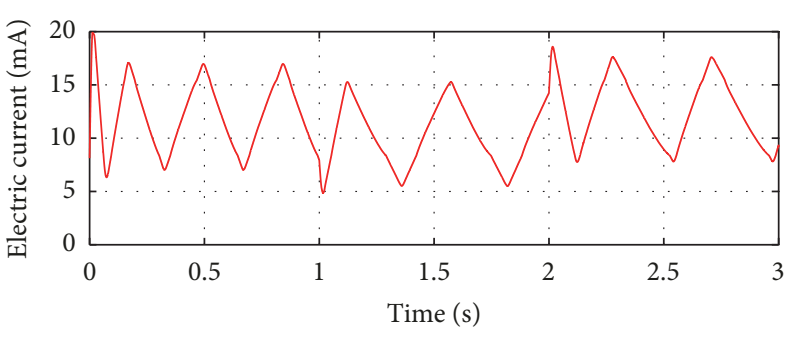

_ Control action

(a) Stem position chart

(b) Control signal chart

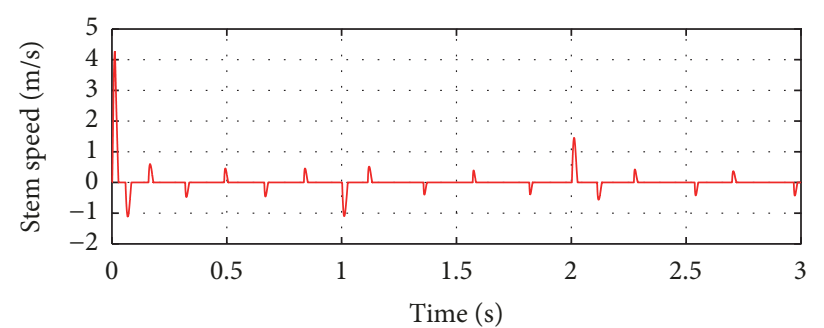

(c) Speed chart

FIgURE 5: Simulation 2. 


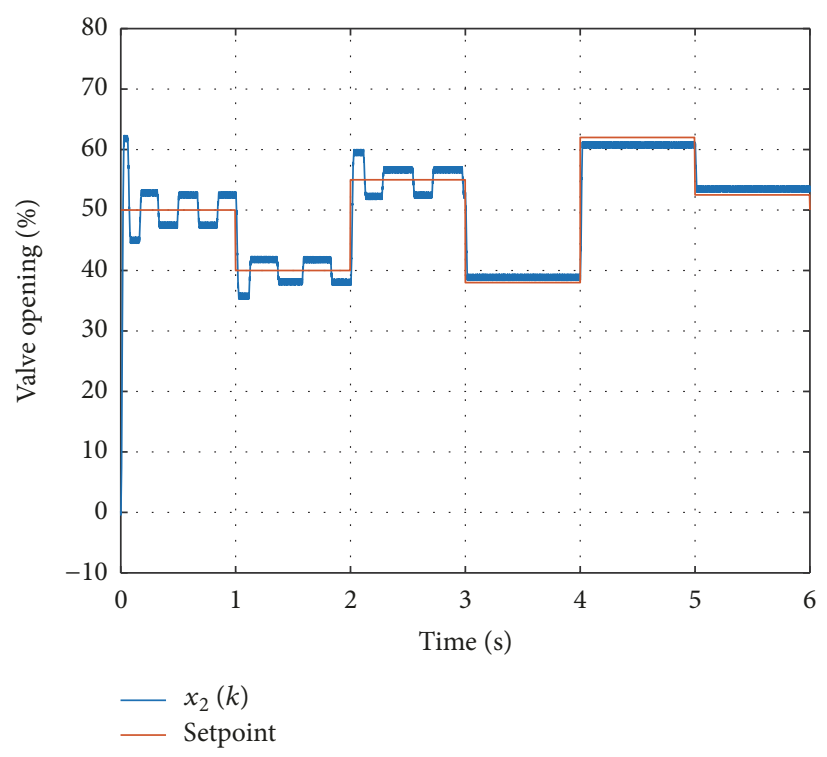

(a) Stem position chart

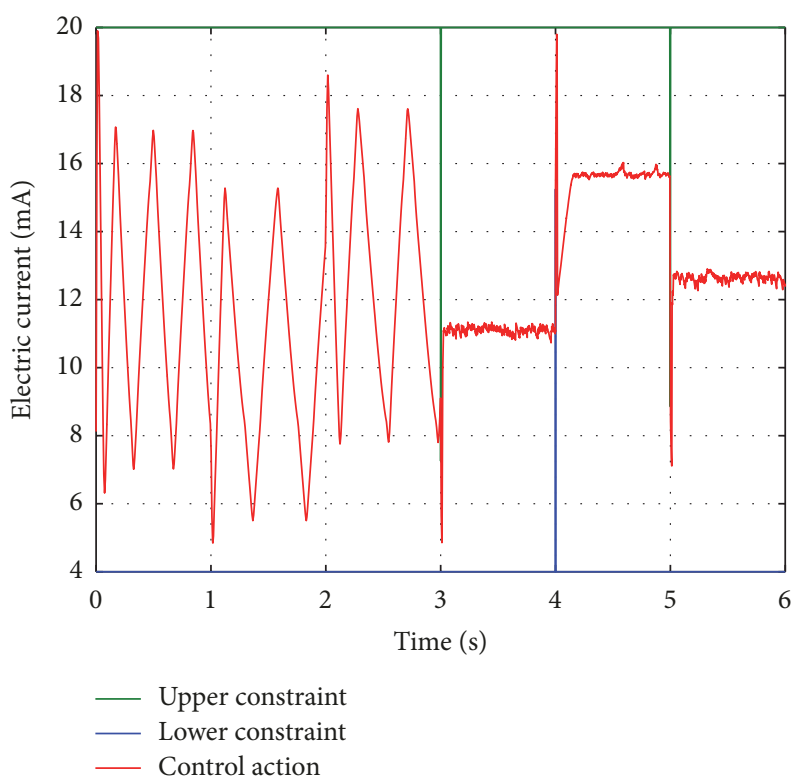

(b) Control signal chart

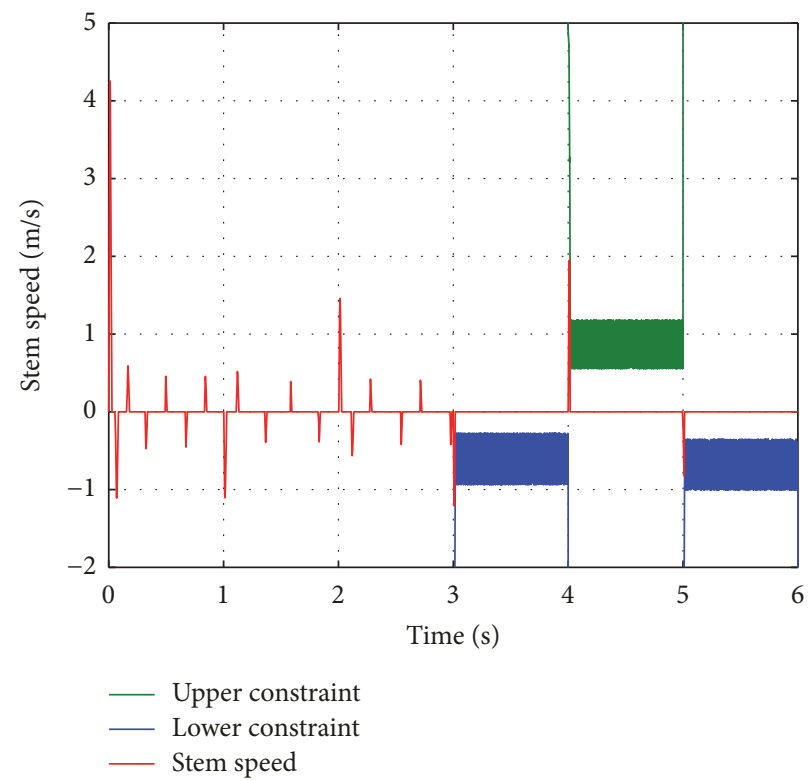

(c) Speed chart

FIGURE 6: Simulation 3.

by the fact that this is inherent to the valve. This decision resets the models parameters described in (21), which results in a system with limit cycle behavior, because there is no damping effect on the valve dynamics.

\subsection{NMPC Applied in the Presence of Stiction Disturbance} $\left(F_{s}=1707.7 \mathrm{~N}, F_{c}=1423 \mathrm{~N}, F_{v}=612.9 \mathrm{~N}\right)$. In simulation 2 , it becomes evident that the inclusion of the static and Coulomb friction components in the valve dynamics reduces the standard predictive controller effectiveness. In the previous simulation, the same controller was able to stabilize the frictionless system and drive the valve output to the correct reference. However, in the present simulation conditions, stick-slip movements that characterize the stiction disturbance continuously occur, presenting oscillations in the valve output trajectory (Figure 5(a)), preventing the error from being satisfactorily treated. In Figure 5(b), it is possible to see the oscillatory behavior of the control signal due to the aggressive tuning of the controller plus the presence of the frictional forces in the model. This behavior can also be seen in the speed peaks presented in Figure 5(c), as a result of the inversions of the stem movement. These inversions in the movement are a reflection of the attempts by the controller to bring the valve to the reference position. 


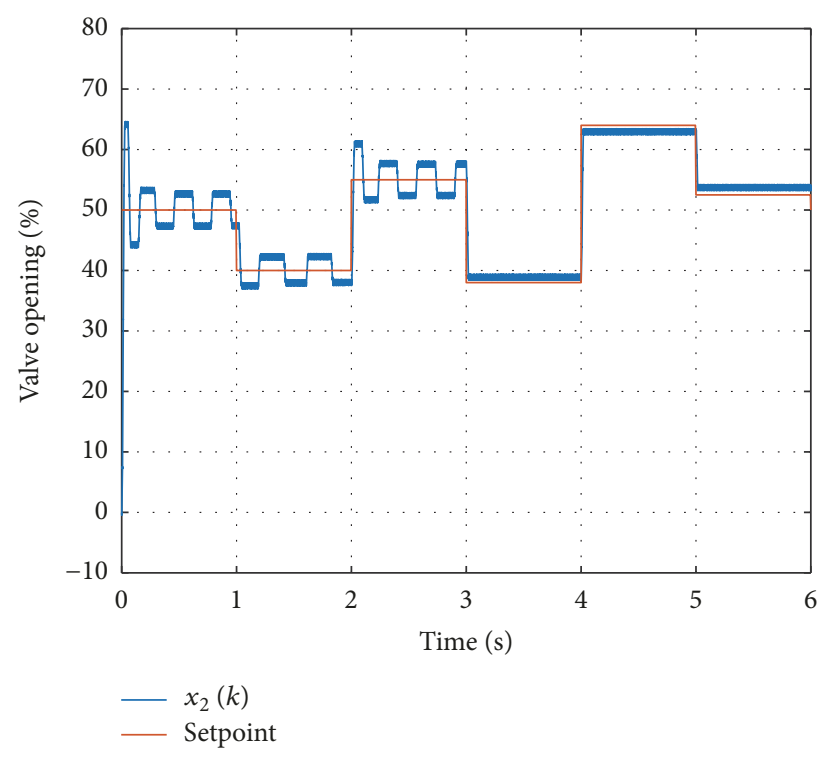

(a) Stem position chart

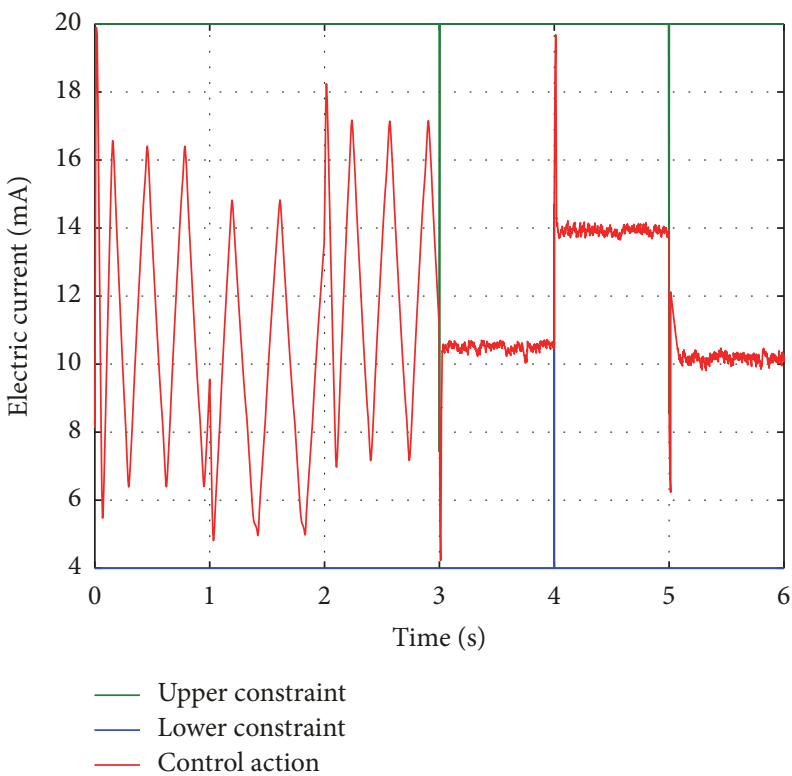

(b) Control signal chart

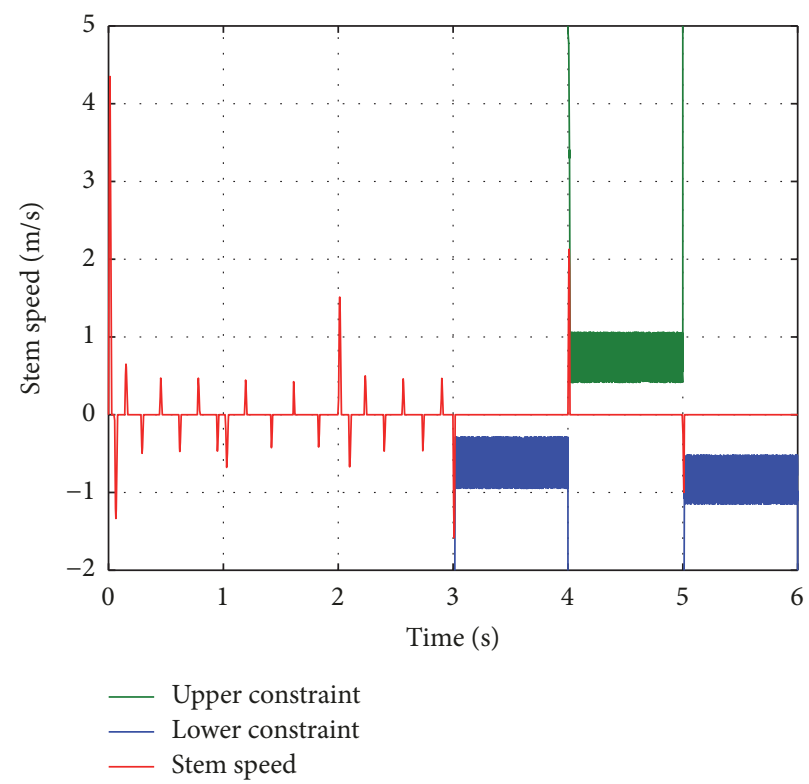

(c) Speed chart

FIGURE 7: Simulation 4.

6.3. NMPC with Proposed Stiction Treatment Strategy $\left(F_{s}=\right.$ $\left.1707.7 \mathrm{~N}, F_{c}=1423 \mathrm{~N}, F_{v}=612.9 \mathrm{~N}\right)$. Through the observation of the results presented by simulation 3 in Figures 6(a), 6(b), and 6(c), one can notice that, up until the instant of 3 seconds, the valve has a similar behavior to that seen in simulation 2. This is because the stiction table is empty and the controller does not know the speed that drives the valve to the desired reference, which causes periodical oscillations of the position variable around the reference. Between the $3 \mathrm{rd}$ and 4th seconds, the table is not empty and the method of control has learned from 3 reference changes that occurred between the 1st and 2nd seconds and the average speed required to drive the valve to the desired reference. The input signal value needed to release the valve grasped is also registered in the table. This information is used to update the input constraints in the predictive control formulation that forces its detachment potentially sooner. In Figures 6(b) and 6(c), we observe that, from the third second, the control signal and the stem speed stabilize, which become almost constant with peaks in the reference transitions.

The green and blue lines in Figures 6(b) and 6(c) represent minimum and maximum constraints on the controller, respectively, and these constraints vary as a function of the error. 


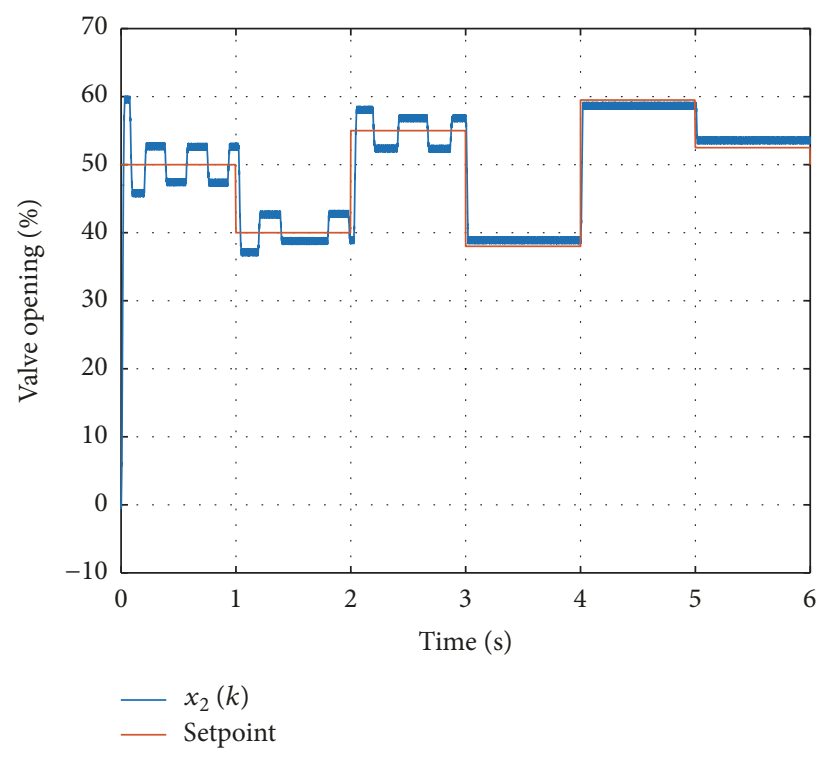

(a) Stem position chart

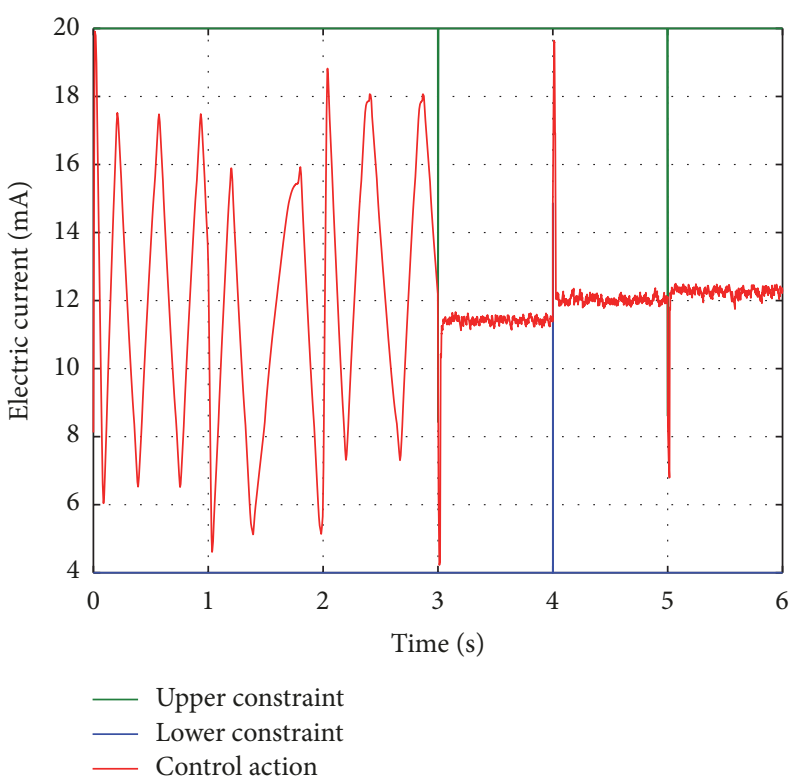

(b) Control signal chart

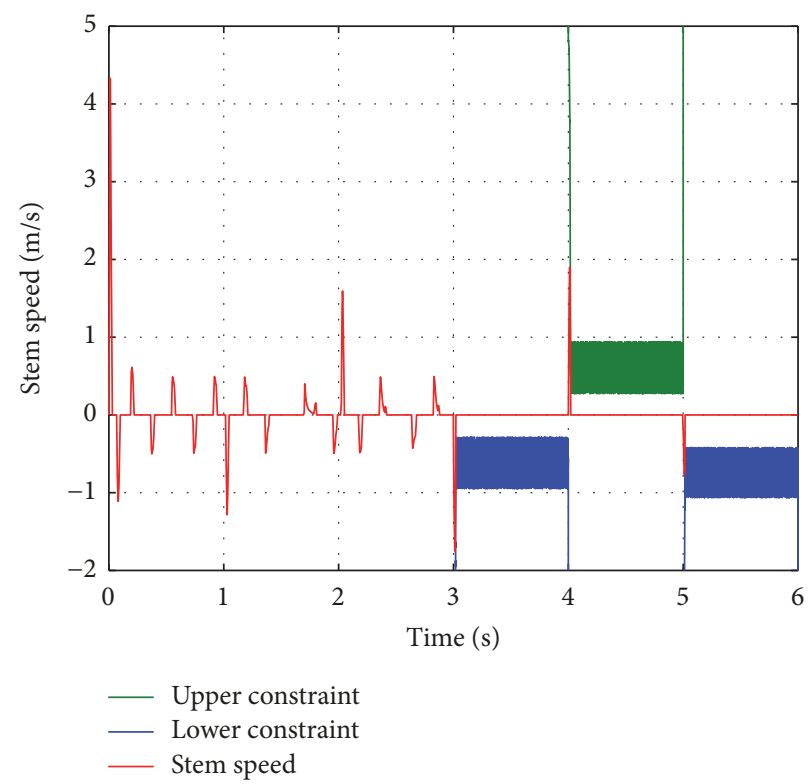

(c) Speed chart

FIGURE 8: Simulation 5.

6.4. NMPC Proposed System with Stiction and Degradation in the Spring Constant $10 \%\left(F_{s}=1707.7 \mathrm{~N}, F_{c}=1423 \mathrm{~N}, F_{v}=\right.$ $612.9 \mathrm{~N})$. Simulation 4 shows that even with a change in the parameter $K_{m}$ (spring constant), which consequently causes a mismatch between the valve model and the controller model, the system continues to compensate the stiction, since the online learning and the minimum speed (present in the stiction table) define where the valve will stop.

Through the analysis of the position charts in simulation 4 , it is possible to notice that the softer spring causes oscillations from 1 to 3 seconds with higher energy when compared to the same chart in simulation 3 , since the decrement in the $K$ value has an opposite action to the pressure force relative to the control action $u$. Even so, the stiction compensation works satisfactorily.

6.5. NMPC Proposed System with Stiction and Increased Friction Parameters by $10 \%\left(F_{s}=18784.7 \mathrm{~N}, F_{c}=1565.3 \mathrm{~N}\right.$, $\left.F_{v}=674.19 \mathrm{~N}\right)$. Simulation 5 proves that even with changes in all the parameters related to the stiction disturbance, which consequently cause mismatching between the valve model and the controller model, the system continues to compensate for the stiction since the learning is online and it will be the minimum speed valve in the stiction table which defines where it will stop. 
Through the position charts for simulation 5, in Figures $8(\mathrm{a}), 8(\mathrm{~b})$, and 8 (c), it is possible to conclude that the increase in the stiction parameters causes the oscillations from 1 to 3 seconds on the system to be lower than the simulation 3 since the increase in the friction effect has a contrary action to the pressure force in comparison to the control action. Even under these conditions, the stiction compensation scheme works satisfactorily.

There are two main reasons that steady state error is not null: (1) the adjustment of the $\alpha$ and $\beta$ constants and (2) the average velocity recorded in the table $\left(v_{\mathrm{Ag}}\right)$ and parameters of (22). In the case of $v_{\mathrm{Ag}}$, the small value associated with it due to statistical bias, because there are few samples of speed in the table. An alternative solution to this problem is the empirical definition of the slightly larger parameters of $\alpha$ and $\beta$ and the increase of the statistical information of the speeds as a function of the position of the stem. Furthermore, in Figures 6(c), 7(c), and 8(c), it is possible to observe, from the instant of $3 \mathrm{~s}$, that the upper (green line) and lower (blue line) constraints of $x_{2}$ show an irregular behavior. This is due to the fact that the state variable $x_{2}$ is a derivative of the position variable $x_{1}$, amplifying the measurement noise.

\section{Conclusion}

This paper presented a system that deals with static friction in electropneumatic valves, one of the most serious problems encountered in these devices, which causes oscillations and consequent wear of the mobile components and production losses. The main idea for the construction of the system is based on a simple principle, which consists of the use of stiction in its favor, instead of trying to compensate it, as presented in previous papers. The strategy adopted uses a nonlinear predictive controller with flexible constraints. The choice of the control strategy is justified by the presence of severe nonlinearities and the presence of the stick-slip phenomenon in the dynamics of the valve, even more so in the latter case which, because of its stochastic nature, requires different values of the control signal to overcome static friction in the same specific position (setpoint), which is not possible with traditional model-based controllers. As an alternative solution to the studied problem, it is proposed to implement a nonlinear and iterative predictive control system for static friction compensation that, associated with a database, reduces the degeneration of the valve positioning system caused by the stick-slip. We describe briefly some of the main linear displacement valve parts used in the industry, as well as their operation and the mathematical models used in the tests (classic model and Karnopp model) of the methodology of control proposed by this paper. In the simulations graphs (Figures 4 and 5), it was observed that the existence of stiction significantly affects the control and that the oscillatory effect was eliminated by the controller presenting a small stationary error (Figures 6, 7, and 8). The controller presented enough robustness to deal with variations in stiction and spring parameters (Figures 7 (b) and $8(\mathrm{~b})$ ), which may occur in valves exposed to temperature variations and by the natural wear of the spring and sealing gaskets because of us.

\section{Conflicts of Interest}

The authors declare that they have no conflicts of interest.

\section{References}

[1] M. S. Choudhury, M. Jain, and S. L. Shah, "Stiction definition, modelling, detection and quantification," Journal of Process Control, vol. 18, no. 3-4, pp. 232-243, 2008.

[2] C. Garcia, "Comparison of friction models applied to a control valve," Control Engineering Practice, vol. 16, no. 10, pp. 1231-1243, 2008.

[3] R. Srinivasan and R. Rengaswamy, "Stiction compensation in process control loops: a framework for integrating stiction measure and compensation," Industrial \& Engineering Chemistry Research, vol. 44, no. 24, pp. 9164-9174, 2005.

[4] Q. P. He and J. Wang, "Valve stiction quantification method based on a semiphysical valve stiction model," Industrial \& Engineering Chemistry Research, vol. 53, no. 30, pp. 12 010-12 022, 2014.

[5] A. Ingimundarson and T. Hägglund, "Performance comparison between PID and dead-time compensating controllers," Journal of Process Control, vol. 12, no. 8, pp. 887-895, 2002.

[6] J. Richalet, A. Rault, J. L. Testud, and J. Papon, "Model predictive heuristic control," Automatica, vol. 14, no. 5, pp. 413-428, 1978.

[7] C. E. Garcia, D. M. Prett, and M. Morari, "Model predictive control: theory and practice a survey," Automatica, vol. 25, no. 3, pp. 335-348, 1989.

[8] S. J. Qin and T. A. Badgwell, "A survey of industrial model predictive control technology," Control Engineering Practice, vol. 11, no. 7, pp. 733-764, 2003.

[9] J. M. Maciejowski, "Predictive control with constraints," International Journal of Adaptive Control and Signal Processing, vol. 17, pp. 261-262.

[10] E. F. Camacho and C. Bordons, Model Predictive Control, Springer, London, UK, 2007.

[11] T. Shen, K. Tamura, N. Henmi, and T. Nakazawa, "Robust model following controller applied to positioning of pneumatic control valve with friction," in Proceedings of the 1998 International Conference on Control Applications, pp. 512-516, Trieste, Italy.

[12] M. Shoukat Choudhury, N. Thornhill, and S. Shah, "Modelling valve stiction," Control Engineering Practice, vol. 13, no. 5, pp. 641-658, 2005.

[13] A. B. Fontes, C. E. Dorea, and M. R. da S. Garcia, "An iterative algorithm for constrained MPC with stability of bilinear systems," in Proceedings of the 16th Mediterranean Conference on Control and Automation, pp. 1526-1531, Ajaccio, France, June 2008.

[14] A. Kayihan and F. J. Doyle, "Friction compensation for a process control valve," Control Engineering Practice, vol. 8, no. 7, pp. 799-812, 2000.

[15] T. Hägglund, "A friction compensator for pneumatic control valves," Journal of Process Control, vol. 12, no. 8, pp. 897-904, 2002.

[16] L. Z. X. Ivan and S. Lakshminarayanan, "A new unified approach to valve stiction quantification and compensation," Industrial \& Engineering Chemistry Research, vol. 48, no. 7, pp. 3474-3483, 2009.

[17] H. Olsson, Control Systems with Friction, Department of Automatic Control Lund Institute of Technology, 1996. 
[18] A. Stenman, F. Gustafsson, and K. Forsman, "A segmentationbased method for detection of stiction in control valves," International Journal of Adaptive Control and Signal Processing, vol. 17, no. 7-9, pp. 625-634, 2003.

[19] M. Kano, H. Maruta, H. Kugemoto, and K. Shimizu, "Practical model and detection algorithm for valve stiction," IFAC Proceedings Volumes, vol. 37, no. 9, pp. 859-864, 2004.

[20] Q. P. He, J. Wang, M. Pottmann, and S. J. Qin, "A curve fitting method for detecting valve stiction in oscillating control loops," Industrial \& Engineering Chemistry Research, vol. 46, no. 13, pp. 4549-4560, jun 2007.

[21] D. Karnopp, "Computer simulation of stick-slip friction in mechanical dynamic systems," Journal of Dynamic Systems, Measurement, and Control, vol. 107, no. 1, pp. 100-103, 1985.

[22] B. Armstrong-Hélouvry, P. Dupont, and C. C. de Wit, "A survey of models, analysis tools and compensation methods for the control of machines with friction," Automatica, vol. 30 , no. 7 , pp. 1083-1138, 1994.

[23] C. Canudas de Wit, H. Olsson, K. J. Astrom, and P. Lischinsky, "A new model for control of systems with friction," IEEE Transactions on Automatic Control, vol. 40, no. 3, pp. 419-425, 1995.

[24] B. C. Silva and C. Garcia, "Comparison of stiction compensation methods applied to control valves," Industrial \& Engineering Chemistry Research, vol. 53, no. 10, pp. 3974-3984, 2014. 


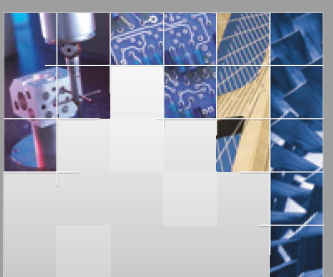

\section{Enfincering}
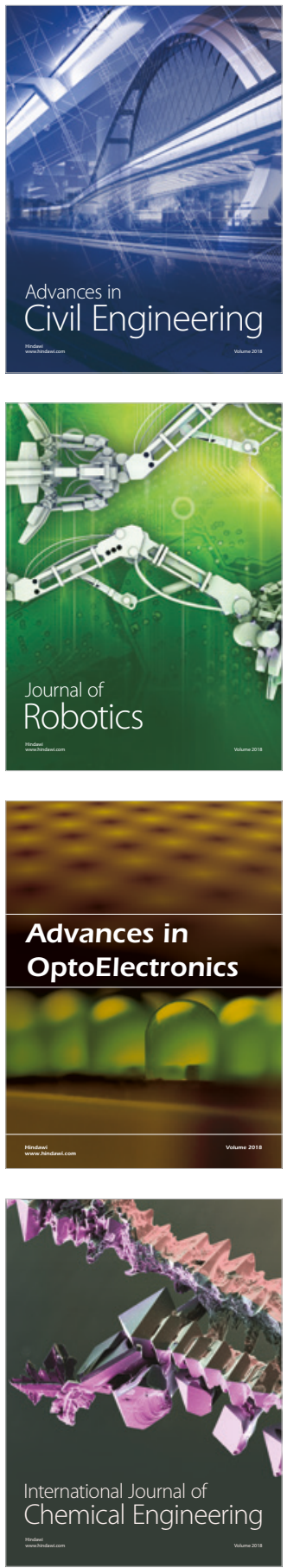

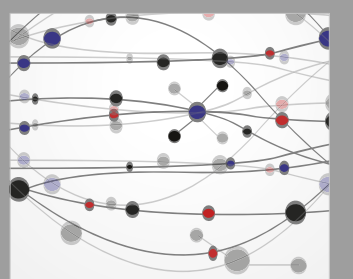

\section{Rotating \\ Machinery}

The Scientific World Journal

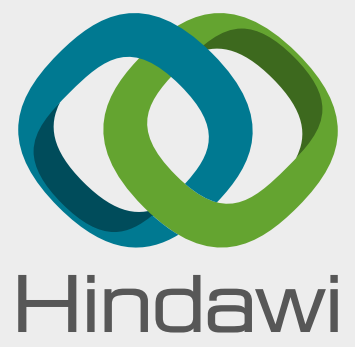

Submit your manuscripts at

www.hindawi.com
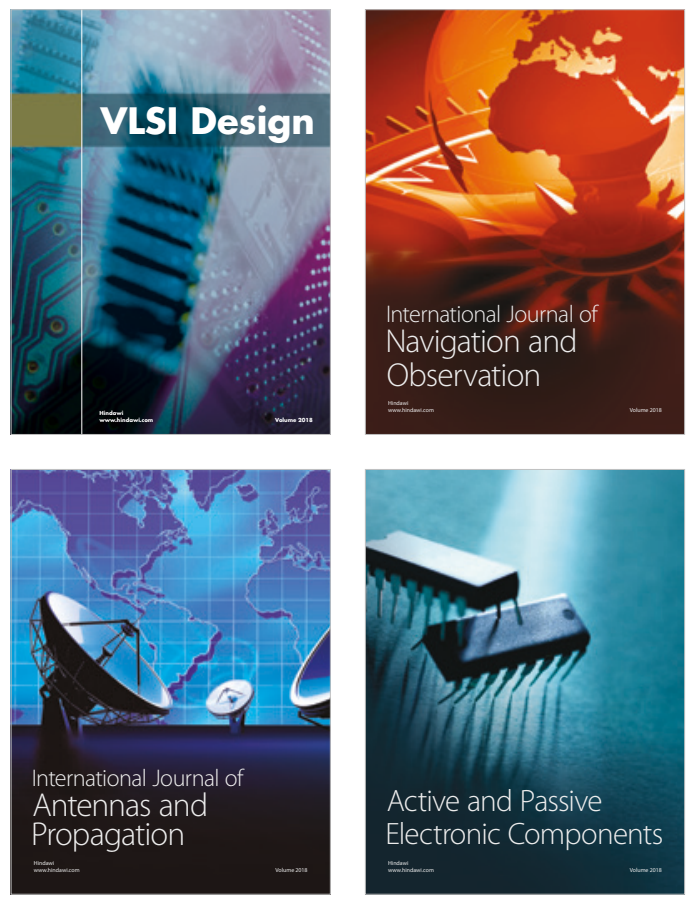
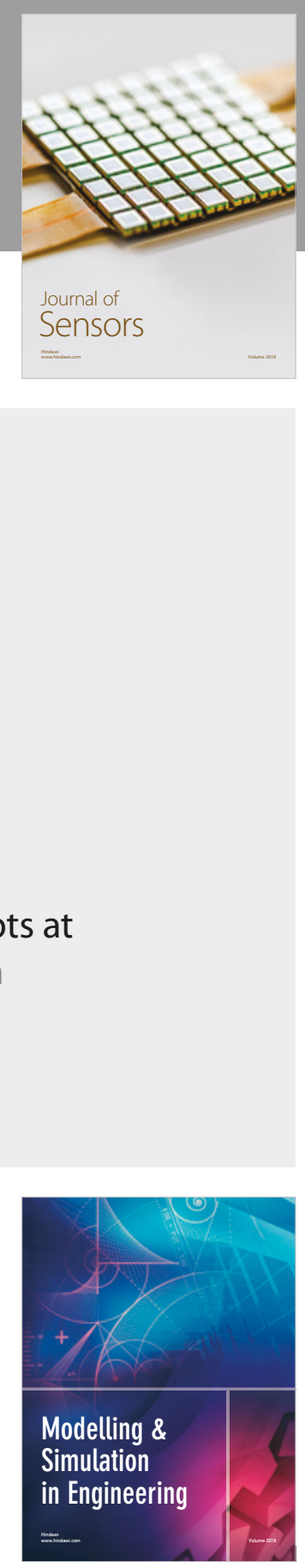

\section{Advances \\ Multimedia}
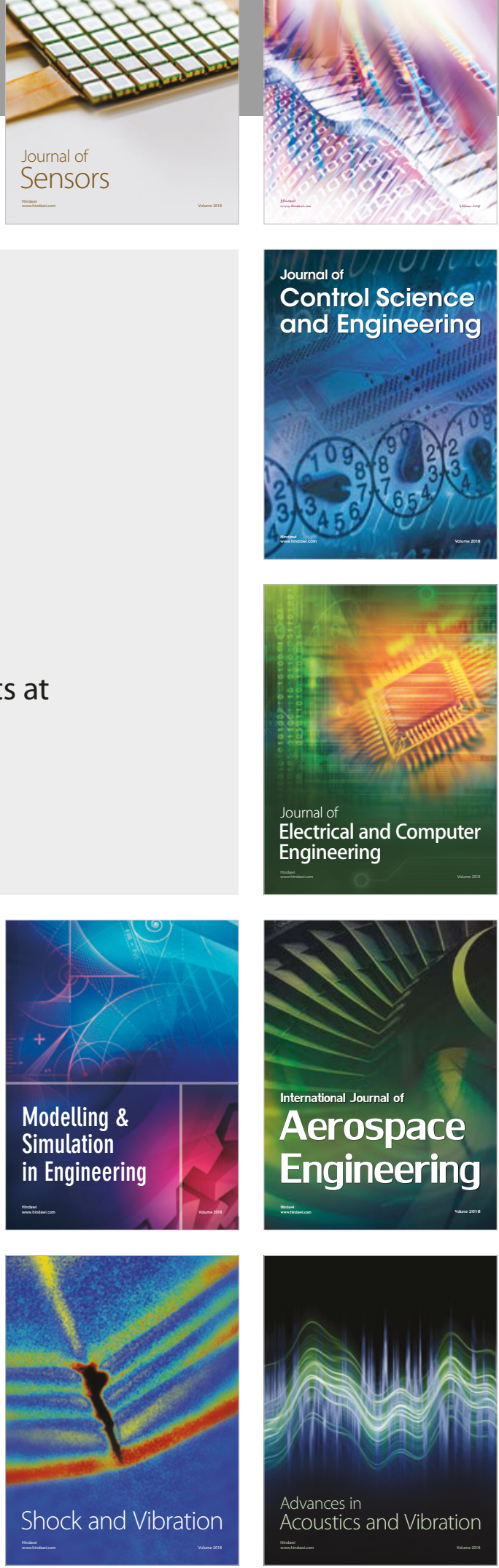